\title{
ИЗМЕНЕНИЕ СУТОЧНОГО РИТМА СОДЕРЖАНИЯ ИНГИБИРУЮЩЕГО ЛЕЙКЕМИЮ ФАКТОРА В КРОВИ БОЛЬНЫХ ЭССЕНЦИАЛЬНОЙ ГИПЕРТЕНЗИЕЙ
}

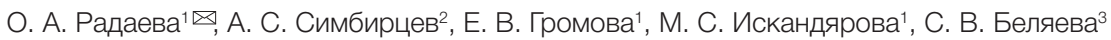

${ }_{1}^{1}$ Медицинский институт Национального исследовательского Мордовского государственного университета имени Н. П. Огарева, Саранск, Россия

2 Государственный научно-исследовательский институт особо чистых биопрепаратов Федерального медико-биологического агентства, Санкт-Петербург, Россия

${ }^{3}$ Санаторно-курортный комплекс «Северокавказский», Пятигорск, Россия

Ингибирующий лейкемию фактор (LIF) обладает неоднозначными физиологическими эффектами при эссенциальной артериальной гипертензии (ЭАГ). Опровергается постулат об идентичности STAT3-сигнализации в клетках миокарда и эндотелиальных/гладкомышечных клетках, что значимо при длительном воздействии цитокинов-лигандов gр 130 (в частности LIF). При этом отсутствуют данные о суточных характеристиках содержания LIF в крови с потенциальными вторичными изменениями его патофизиологических эффектов. Целью исследования было проанализировать особенности суточных ритмов содержания LIF в сыворотке периферической крови больных ЭАГ II стадии, определенного в пяти временны́х точках в зависимости от применения гипотензивной терапии и частоты развития осложнений в последующие 5 лет наблюдения. У 60 больных ЭАГ II стадии иммуноферментным методом определяли уровни LIF в сыворотке периферической крови в 8.00, 14.00, 20.00, 2.00, 8.00 ч до и после года гипотензивной терапии. Выявленные закономерности суточного ритма содержания LIF в сыворотке крови больных ЭАГ II стадии в виде увеличения в 20.00 ч на 15\% (p < 0,001) и выше с максимальным ростом в 2.00 ч на 22\% ( $<$ 0,001) и более при сравнении с индивидуальным уровнем в 8.00 ч можно характеризовать как патологические, а их сохранение после года гипотензивной терапии служит признаком прогредиентного течения гипертензии с повышением риска развития сердечно-сосудистых осложнений (инфаркт миокарда, острое нарушение мозгового кровообращения) в 6 раз

Ключевые слова: LIF, ингибирующий лейкемию фактор, суточный ритм цитокинов, эссенциальная гипертензия

Вклад авторов: О. А. Радаева - разработка дизайна исследования, анализ результатов, формулировка выводов, осормление рукописи; А. С. Симбирцев - формулирование цели исследования, корректирование выводов и итогового варианта рукописи; Е. В. Громова - разработка дизайна исследования, проведения лабораторного этапа исследования, оформление рукописи; М. С. Искандярова - работа с литературой, контроль забора материала, динамическое наблюдение за пациентами, работа над первым вариантом рукописи; С. В. Беляева - работа с литературой, контроль забора материала и участие в наблюдении за пациентами.

Соблюдение этических стандартов: исследование одобрено этическим комитетом Мордовского государственного университета имени Н. П. Огарева (протокол № 12 от 14 декабря 2008 г.). Все пациенты подписали добровольное информированное согласие. Получение биологического материала для исследования (кровь) производили с учетом положений Хельсинской декларации ВМА (2008 г.) и протокола Конвенции Совета Европы о правах человека и биомедицине (1999) с учетом дополнительного протокола к Конвенции по правам человека и биомедицине в области биомедицинских исследований (2005).

$\varangle$ Для корреспонденции: Ольга Александровна Радаева ул. Ульянова, д. 26а, г. Саранск, 430000; vtlbwbyf_79@mail.ru

Статья получена: 11.03.2020 Статья принята к печати: 25.03.2020 Опубликована онлайн: 28.03.2020

DOI: $10.24075 /$ vrgmu.2020.017

\section{CIRCADIAN RHYTHMS OF LEUKEMIA INHIBITORY FACTOR IN THE BLOOD OF PATIENTS WITH ESSENTIAL HYPERTENSION}

\author{
Radaeva $\mathrm{OA}^{1} \otimes$, Simbirtsev $\mathrm{AS}^{2}$, Gromova EV1, Iskandiarova MS ${ }^{1}$, Belyaeva SV³ \\ ${ }^{1}$ Institute of Medicine, National Research Mordovia State University, Saransk, Russia \\ 2 State Research Institute of Highly Pure Biopreparations, FMBA, Saint Petersburg, Russia \\ ${ }^{3}$ North Caucasus Health Center, Pyatigorsk, Russia
}

Leukemia inhibitory factor (LIF) exerts multidirectional effects in the setting of essential hypertension (EH). There is a mounting body of evidence refuting the postulate about identical STAT3 signaling in cardiomyocytes and endothelial/smooth muscle cells, which is important in the situation of extended exposure to gp 130 ligands (LIF in particular). At the same time, there are no reports on the circadian dynamics of peripheral blood LIF concentrations and possible secondary changes to the pathophysiological effects of this cytokine. This study aimed to analyze the circadian dynamics of LIF concentrations in the peripheral blood serum measured at 5 different time points in patients with stage II EH in the presence/absence of antihypertensive therapy and their relationship with the frequency of complications developing within a 5-year follow-up. Blood serum LIF was measured in 60 patients with stage II EH using ELISA at 8:00, 14:00, 20:00, 2:00, and 8:00 o'clock before putting the patients on antihypertensive therapy and one year after its onset. The identified patterns of diurnal LIF concentrations (a rise by $\geq 15 \%$ at 20:00, $p<0.001$; a further rise by $\geq 22 \%$ peaking at 2:00, $p<0.001$ relative to the values at 8:00) can be regarded as pathologic; their persistence after one year of antihypertensive therapy is a sign of EH progression and puts the patients at 6-fold risk for cardiovascular complications, including myocardial infarction and acute cerebrovascular events.

Keywords: LIF, leukemia inhibitory factor, cytokine circadian rhythms, essential hypertension

Author contribution: Radaeva OA designed the study, analyzed the results, formulated the conclusions and wrote the manuscript; Simbirtsev AS formulated the objective of the study, revised its conclusions and the manuscript itself; Gromova EV designed the study, carried out laboratory tests, and contributed to writing the manuscript; Iskandiarova MS analyzed the literature, supervised blood collection, followed up with the patients, contributed to writing the manuscript; Belyaeva SV analyzed the literature, supervised blood collection, followed up with the patients.

Compliance with ethical standards: the study was approved by the Ethics Committee of National Research Mordovia State University (Protocol No. 12 dated December 14, 2008). Written informed consent was obtained from all study participants. Blood samples were collected in compliance with the Declaration of Helsinki (2008), the protocol of European Convention on Human Rights and Biomedicine (1999) and the additional protocol to the Convention on Human Rights and Biomedicine concerning Biomedical Research (2005).

$\triangle$ Correspondence should be addressed: Olga A. Radaeva

Ulianova, 26a, Saransk, 430000; vtlbwbyf_79@mail.ru

Received: 11.03.2020 Accepted: 25.03.2020 Published online: 28.03.2020

DOI: $10.24075 /$ brsmu.2020.017 
Ингибирующий лейкемию фрактор (LIF) обладает разнообразными физиологическими эффектами за счет локализации специфичных рецепторов LIF на мембранах эндотелиоцитов, моноцитов, нейронов и др. [1], в физиологически значимых количествах [2]. Сигнальный путь LIF через JAK/STAT (Janus kinase/signal transducer and activator of transcription), MAPK (mitogen-activated protein kinases) и PI3K (phosphoinositide-3-kinases) является стабильным, но реализует противоположные эффекты действия в различных типах клеток, включая и стимулирование, и блокирование дифференцировки и выживания этих клеток. Вызывают дискуссию результать о влиянии LIF на стенку сосудов при эссенциальной артериальной гипертензии (ЭАГ), так как механизм активации STAT3 редокс-зависим [3] и меняет вектор при хроническом повышении АД, искажая эффекты LIF. Опровергается постулат об идентичности STAT3сигнализации в клетках миокарда и эндотелиальных/ гладкомышечных клетках, что значимо при длительном воздействии цитокинов-лигандов gр 130 (в частности LIF) [4]. Ряд научных направлений демонстрирует зависимость компонентов прогрессирования ЭАГ, формирования осложнений от времени суток [5], доказывая значимость научного поиска не только количественных изменений цитокинов, но и вариантов их суточных ритмов синтеза.

Целью исследования было проанализировать особенности суточных ритмов содержания LIF в сыворотке периферической крови больных ЭАГ ІІ стадии, измеренного в пяти временных точках $(8.00,14.00,20.00$, 2.00 и 8.00 4), в зависимости от применения гипотензивной терапии и частоты развития осложнений в последующие 5 лет наблюдения.

\section{ПАЦИЕНТЫ И МЕТОДЫ}

В рамках исследования на тему «Цитокины в патогенезе ЭАГ», выполненного в период с 2008 по 2019 г. на базе Медицинского института НИ МГУ им. Н. П. Огарева, Регионального сосудистого центра ГБУЗ РМ «Республиканская клиническая больница № 4», в 2014 г. сформирована дополнительная группа из 60 больных ЭАГ II стадии (30 мужчин, 30 женщин) для изучения в течение суток изменения содержания LIF в сыворотке крови.
Критерии включения пациентов в исследование: ЭАГ ॥ стадии, 1955-1956 год рождения, анамнез заболевания 10-14 лет; отсутствие гипотензивной терапии в начале исследования, наличие сопоставимой терапии в течение следующего года (и-АПФ \pm диуретик) с достижением «целевого» уровня АД (в соответствии с российскими рекомендациями «Диагностика и лечение артериальной гипертензии», 2010) [6]; уровни общего холестерина менее 5,0 ммоль/л, ЛПНП менее 3,0 ммоль/л, ЛПВП более 1,0 ммоль/л, триглицеридов менее 1,7 ммоль/л, глюкозы менее 5,5 мг/дл, ИМТ менее 30 кг/м²; сопоставимый риск развития осложнений ЭАГ. Критерии исключения: наличие ассоциированных клинических состояний; сахарный диабет 1-го или 2-го типов, аутоиммунные, аллергические заболевания, симптоматическая артериальная гипертензия. Группа сопоставления - условно здоровые 30 человек (15 мужчин, 15 женщин) с САД от 100 до 130 мм рт. ст. и ДАД 70-89 мм рт. ст., сопоставимые по возрасту и основным биохимическим параметрам с пациентами группы наблюдения.

Забор крови (по 2 мл) проводили до начала гипотензивной терапии (2014 г.) и через год терапии (2015 г.) в 8.00, 14.00, 20.00, 2.00, 8.00 (интервал без приема пищи составлял не менее 6 4). Временные отрезки были выбраны на основе данных пилотного исследования, включающего забор крови в 7.00, 8.00, 10.00, 12.00, 14.00, 16.00, 18.00, 20.00, 22.00, 00.00, 2.00, 4.00, 6.00, 7.00, 8.00 у 7 человек. Время от забора крови до замораживания сыворотки составляло 60 мин. Определение LIF в сыворотке крови проводили иммуноферментным методом с использованием тест-систем (Bender MedSystems; США).

С 2014 по 2019 г. проводили ежегодный телефонный опрос участников исследования для регистрации осложнений (инфаркт миокарда (ИМ), острое нарушение мозгового кровообращения (ОНМК), транзиторное нарушение мозгового кровообращения (ТНMК)) в период наблюдения с последующим подтверждением диагноза на основе данных истории болезни: клинических и диагностических (ЭКГ, ЭХО-КГ, теста на уровень тропонина, данных компьютерной томографии головного мозга).

Статистическую обработку данных проводили с помощью пакетов прикладных программ Statistica 10.0 (Stat Soft; США). Нормальность распределения

Таблица 1. Содержание LIF (пг/мл) в сыворотке периферической крови больных ЭАГ ІІ стадии в 8.00, 14.00, $20.00,2.00$ и 8.00 ч (следующие сутки) в зависимости от применения гипотензивных препаратов и развития сердечно-сосудистых осложнений в следующие 5 лет наблюдения (Ме (Q25\%-Q75\%))

\begin{tabular}{|c|c|c|c|c|c|c|}
\hline Группы & & $\begin{array}{c}8.00 \\
\text { (первые сутки) }\end{array}$ & $\begin{array}{c}14.00 \\
\text { (первые сутки) }\end{array}$ & $\begin{array}{c}20.00 \\
\text { (первые сутки) }\end{array}$ & $\begin{array}{c}2.00 \\
\text { (вторые сутки) }\end{array}$ & $\begin{array}{c}8.00 \\
\text { (вторые сутки) }\end{array}$ \\
\hline $\begin{array}{l}\text { Больные ЭАГ (до терапии) } \\
n=60 \text { (человек) }\end{array}$ & $a$ & $7,51[6,58-8,34]$ & $7,58[6,47-8,41]$ & $\begin{array}{c}9,02[7,52-9,73] \\
\quad * 8.00,14.00\end{array}$ & $\begin{array}{c}10,1[9,44-11,8] \\
* 8.00,14.00,20.00 \\
\end{array}$ & $\begin{array}{l}7,53[6,65-8,22] \\
{ }^{*} 20.00,2.00\end{array}$ \\
\hline \multirow[t]{2}{*}{ Группа сравнения (здоровые), $n=30$} & $b$ & $1,41[1,02-1,83]$ & $1,38[1,04-1,79]$ & $1,45[1,06-1,78]$ & $1,42[1,03-1,81]$ & $1,37[0,99-1,79]$ \\
\hline & & $p(b-a)<0,001$ & $p(b-a)<0,001$ & $p(b-a)<0,001$ & $p(b-a)<0,001$ & $p(b-a)<0,001$ \\
\hline \multirow[t]{2}{*}{$\begin{array}{l}\text { Больные ЭАГ (после года терапии), } \\
n=60, \text { из них: }\end{array}$} & $c$ & $7,54[6,57-8,38]$ & $7,61[6,53-8,44]$ & $\begin{array}{l}8,95[7,63-9,58] \\
\quad * 8.00,14.00 \\
\end{array}$ & $\begin{array}{l}7,62[6,84-8,63] \\
\quad 18.00 * 20.00 \\
\end{array}$ & $\begin{array}{c}7,58[6,62-8,31] \\
* 20.00 \\
\end{array}$ \\
\hline & & $\begin{array}{l}p(c-a)>0,05 \\
p(c-b)<0,001\end{array}$ & $\begin{array}{c}p(c-a)>0,05 \\
p(c-b)<0,001\end{array}$ & $\begin{array}{c}p(c-a)>0,05 \\
p(c-b)<0,001\end{array}$ & $\begin{array}{l}p(c-a)<0,001 \\
p(c-b)<0,001\end{array}$ & $\begin{array}{l}p(c-a)<0,05 \\
p(c-b)<0,001\end{array}$ \\
\hline $\begin{array}{l}\text { Следующие } 5 \text { лет наблюдения - } \\
\text { ИМ, ОНМК, } n=15\end{array}$ & $c 1$ & $7,66[6,68-8,22]$ & $7,59[6,49-8,37]$ & $\begin{array}{l}9,01[7,56-9,61] \\
\quad * 8.00,14.00\end{array}$ & $\begin{array}{c}9,69[8,91-9,98] \\
* 8.00,14.00,20.00\end{array}$ & $\begin{array}{l}7,72[6,71-8,17] \\
\quad * 20.00,2.00\end{array}$ \\
\hline \multirow[t]{2}{*}{$\begin{array}{l}\text { Следующие } 5 \text { лет - } \\
\text { без осложнений, } n=45\end{array}$} & $c 2$ & $7,58[6,52-8,41]$ & $7,62[6,5-8,42]$ & $\begin{array}{l}8,79[7,98-9,62] \\
\quad * 8.00,14.00\end{array}$ & $\begin{array}{c}7,55[6,93-8,21] \\
* 20.00\end{array}$ & $\begin{array}{c}7,36[6,51-8,24] \\
* 20.00\end{array}$ \\
\hline & & $p(c 2-c 1)>0,05$ & $p(c 2-c 1)>0,05$ & $p(c 2-c 1)>0,05$ & $p(c 2-c 1)<0,001$ & $p(c 2-c 1)>0,05$ \\
\hline
\end{tabular}

Примечание: достоверно при сравнении с указанным временем забора крови или группой $(a, b, c, c 1, c 2) ;{ }^{\star}-p<0,001 ;$; $-p<0,01 ;$; $-p<0,05$. Для сравнения результатов в 8.00, 14.00, 20.00, 2.00 и 8.00 ч внутри группы больных ЭАГ ІІ стадии до терапии - парный $t$-критерий Стьюдента, внутри групп «после года терапии» и здоровых (группа сопоставления) - критерий Уилкоксона, между независимыми группами - критерий Манна-Уитни и между зависимыми группами - критерий Уилкоксона. 
показателей определяли с помощью одновыборочного критерия Колмагорова-Смирнова, что обосновало использование для сравнения результатов, полученных в 8.00, 14.00, 20.00, 2.00, 8.00 ч внутри группы больных ЭАГ II стадии до терапии, парного $t$-критерия Стьюдента, внутри группы «после года терапии» и здоровых (группа сопоставления) - критерия Уилкоксона, между группами критериев Манна-Уитни (при независимых выборках) и Уилкоксона (зависимые выборки). Данные представлены в виде медианы (Ме) и перцентилей $(\mathrm{Q} 0,25-\mathrm{Q0}, 75)$. При разделении групп на подгруппы использовали поправку Бонферрони для множественных сравнений, что подтвердило корректность приведенных в статье данных статистического анализа. Рассчитывали абсолютный и относительный риски развития ИМ, ОНМК с определением 95\%-го ДИ, чувствительности и специфичности. Проводили анализ с использованием точного критерия Фишера (двустороннего) $\varphi$, нормированного значения коэффициента Пирсона (C').

\section{РЕЗУЛЬТАТЫ ИССЛЕДОВАНИЯ}

У больных ЭАГ ॥ стадии при длительности заболевания 10-14 лет без приема гипотензивных препаратов при сравнении со здоровыми (группа сопоставления) зарегистрированы достоверные количественные и качественные различия суточного ритма содержания LIF в сыворотке перисерической крови. Уровни LIF у пациентов с ЭАГ ॥ стадии в 8.00, 14.00, 20.00 и 2.00 в 5-7,5 раза выше $(p<0,001)$, чем у лиц без ЭАГ (табл. 1). Значимым является рост LIF у больных ЭАГ в 20.00 (на 20,1\% (16,724,3\%); $p<0,001$ ), с максимумом в 2.00 (увеличение на 34\% (25,7-43\%); $p<0,001)$, при сравнении с результатами данной группы, полученными в 8.00. Важно отметить, что в группе здоровых не зарегистрировано $(p>0,05)$ динамики показателей в 14.00, 20.00 и 2.00 при сопоставлении с концентрациями в 8.00. После приема гипотензивных препаратов в течение года и достижения целевого уровня АД в соответствии с клиническими рекомендациями РФ (2010) у пациентов не было зарегистрировано снижения LIF в сыворотке крови в 8.00, 14.00, 20.00 ч при сравнении с периодом до терапии $(p<0,01)$, но изменялась суточная динамика содержания цитокина. На фоне гипотензивной терапии с достижением «целевого» АД максимальный рост LIF был зарегистрирован в 20.00, но со снижением в 2.00 (табл. 2), в отличие от периода до терапии. При этом распределение показателей в группе больных на фоне терапии отличалось от распределения Гаусса-Лапласа, что актуализировало анализ индивидуальных суточных графиков больных этой категории для выявления критерия неоднородности. Было выявлено, что 22 пациента, принимающих гипотензивные препараты и достигших целевого АД, сохранили суточные ритмы содержания LIF в крови, характерные для этапа «до гипотензивной терапии» (рост в 20.00 с максимумом в 2.00 и восстановлением уровней в 8.00) (см. табл. 2).

Анализ катамнеза группы на фоне гипотензивной терапии и частичного восстановления суточных закономерностей LIF (снижение в 2.00) показал, что из 42 пациентов только у 4 больных в течение последующих 5 лет развились ОНМК, ИМ (абсолютный риск осложнений составил 9,5\% (0,63-18,4\%). В группе с сохранением «патологического" суточного ритма содержания LIF в крови (рост в 20.00, пик в 2.00 с восстановлением утренних показателей в 8.00) - у 11 человек из 18 зарегистрированы осложнения (ОНМК, ИМ) абсолютный риск равен 61,1\% (38,6-83,6\%). Отношение рисков двух групп составило 6,41 (2,35-17,5\%), специфичность - 0,84, чувствительность - 0,73, $\varphi-$ 0,0000 (p < 0,05), C' - 0,67 (связь очень сильная).

Таблица 2. Процент отклонения уровней LIF (пг/мл) в сыворотке крови больных ЭАГ II стадии в 14.00, 20.00, 2.00 и 8.00 ч (следующие сутки) от концентрации в 8.00 ч (первые сутки наблюдения) в зависимости от применения гипотензивной терапии и развития осложнений в следующие 5 лет наблюдения (Me (Q25\%-Q75\%))

\begin{tabular}{|c|c|c|c|c|c|}
\hline Группы & & $\begin{array}{c}8.00 \\
\text { (первые сутки) }\end{array}$ & $\begin{array}{c}14.00 \\
\text { (первые сутки) }\end{array}$ & $\begin{array}{c}20.00 \\
\text { (первые сутки) }\end{array}$ & $\begin{array}{c}8.00 \\
\text { (вторые сутки) }\end{array}$ \\
\hline $\begin{array}{l}\text { Больные ЭАГ (до терапии), } \\
n=60\end{array}$ & $a$ & $0,91[-1,67-2,54]$ & $20,1[16,7-24,3] * 14.00$ & $\begin{array}{c}34[25,7-33]{ }^{*} 14.00 \\
20.00\end{array}$ & $\begin{array}{l}1,06[-1,05-3,65] \\
* 20.00,2.00\end{array}$ \\
\hline \multirow[t]{2}{*}{$\begin{array}{l}\text { Группа сравнения (здоровые), } \\
n=30\end{array}$} & $b$ & $-1,08[-2,03-1,19]$ & $2,81[1,93-3,22]$ & $0,98[-0,13-2,03]$ & $1,03[-1,72-2,56]$ \\
\hline & & $p(b-a)>0,05$ & $p(b-a)<0,001$ & $p(b-a)<0,001$ & $p(b-a)>0,05$ \\
\hline \multirow[t]{2}{*}{$\begin{array}{l}\text { Больные ЭАГ (после года терапии), } \\
n=60, \text { из них: }\end{array}$} & $c$ & $0,93[-0,61-2,03]$ & $18,9[15,9-24,1] * 14.00$ & $\begin{array}{l}6,32[2,08-14,4] \\
{ }^{*} 14.00,20.00\end{array}$ & $\begin{array}{l}0,76[-0,45-2,21] \\
\quad * 20.00,2.00\end{array}$ \\
\hline & & $\begin{array}{l}p(c-a)>0,05 \\
p(c-b)>0,05\end{array}$ & $\begin{array}{l}p(c-a)>0,05 \\
p(c-b)<0,001\end{array}$ & $\begin{array}{l}p(c-a)<0,001 \\
p(c-b)<0,05\end{array}$ & $\begin{array}{l}p(c-a)>0,05 \\
p(c-b)>0,05\end{array}$ \\
\hline \multirow[t]{2}{*}{$\begin{array}{l}\text { Следующие } 5 \text { лет наблюдения - ИМ, } \\
\text { ОНМК, } n=15\end{array}$} & $c 1$ & $-0,91[-2,83-1,8]$ & $19,9[15,1-23,8] * 14.00$ & $\begin{array}{l}26,5[22,3-28,1] \\
* 14.00,20.00\end{array}$ & $\begin{array}{l}0,22[-1,35-2,33] \\
\quad * 20.00,2.00\end{array}$ \\
\hline & & $\begin{array}{l}p(c 1-a)>0,05 \\
p(c 1-b)>0,05\end{array}$ & $\begin{array}{l}p(c 1-a)>0,05 \\
p(c 1-b)<0,001\end{array}$ & $\begin{array}{l}p(c 1-a)>0,05 \\
p(c 1-b)<0,001\end{array}$ & $\begin{array}{l}p(c 1-a)>0,05 \\
p(c 1-b)>0,05\end{array}$ \\
\hline \multirow[t]{2}{*}{$\begin{array}{l}\text { Следующие } 5 \text { лет - без осложнений, } \\
n=45\end{array}$} & $c 2$ & $0,52[-0,47-1,67]$ & $18,1[14,8-23,8] * 14.00$ & $\begin{array}{c}2,29[-0,35-5,44] \\
\quad 14.00 * 20.00\end{array}$ & $\begin{array}{l}0,92[-0,62-2,13] \\
* 20.00,2.00\end{array}$ \\
\hline & & $\begin{array}{l}p(c 2-c 1)>0,05 p(c 2-b) \\
>0,05 p(c 2-a)>0,05\end{array}$ & $\begin{array}{l}p(c 2-c 1)>0,05 p(c 2-b) \\
<0,001 p(c 2-a)>0,05\end{array}$ & $\begin{array}{c}p(c 2-c 1)<0,001 \\
p(c 2-b)<0,05 \\
p(c 2-a)<0,001\end{array}$ & $\begin{array}{l}p(c 2-c 1)>0,05 \\
p(c 2-b)>0,05 \\
p(c 2-a)>0,05\end{array}$ \\
\hline
\end{tabular}

Примечание: достоверно при сравнении с указанным временем забора крови или группой $(a, b, c, c 1, c 2)$ * $-p<0,001 ;$ ^ $-p<0,01 ;{ }^{\prime}-p<0,05$. Для сравнения результатов в 8.00, 14.00, 20.00, 2.00 и 8.00 ч внутри группы больных ЭАГ II стадии до терапии - парный $t$-критерий Стьюдента, внутри групп «после года терапии» и здоровых (группа сопоставления) - критерий Уилкоксона, между независимыми группами - критерий Манна-Уитни и между зависимыми группами - критерий Уилкоксона. 


\section{ОБСУЖДЕНИЕ РЕЗУЛЬТАТОВ}

Полученные в рамках исследования данные о повышении содержания LIF в сыворотке периферической крови у больных ЭАГ II стадии по сравнению со здоровыми лицами могут иметь патогенетическое объяснение, обоснованное нарушением процесса интегрирования сигналов LIFR/ CD118 и gр130 на фоне окислительного стресса при ЭАГ, что сказывается на каталитической активности JAK [7] и может стимулировать образование LIF. Зарегистрированный рост в 20.00 с динамическим увеличением в 2.00 у лиц до приема гипотензивных препаратов, а у части пациентов и на фоне терапии с достижением «целевого» АД патогенетически значим, так как есть сообщения, что в эндотелиоцитах LIFзависимая стимуляция STAT3 запускает воспалительный каскад [8] с активацией системы IL1, что в вечернее (20.00) и ночное (2.00) время на фоне снижения активности провоспалительных компонентов (IL1ra, IL10) будет более выражено стимулировать прогрессирование ЭАГ через активацию протеинаргининметилтрансферазы и торможения диметиларгинина с последующим дисбалансом в системе синтеза NO. По нашим данным [9], у пациентов с ЭАГ на фоне увеличения LIF более 7,5 пг/мл определен рост индекса массы миокарда левого желудочка со снижением фракции укороченных средних волокон и достоверной связью с формированием выраженной гипертрофии миокарда левого желудочка концентрического типа. Зарегистрированный патофизиологический процесс позволил выдвинуть гипотезу о повышении риска развития сердечно-сосудистых осложнений у пациентов с сохранением роста LIF в период с 20.00 до 2.00 на фоне терапии, которая подтвердилась в ходе нашего исследования. LIF-индуцированная кардиальная гипертрофия может характеризоваться ранним снижением сократительной способности миокарда (из-за трансмурального изменения кардиомиоцитов) [10, 11].
При этом на начальных этапах патологического процесса повышение LIF является компенсаторно-приспособительным механизмом, стимулирующим сократимость кардиомиоцитов через усиление активности Т-зависимых $\mathrm{Ca}^{2+}$-каналов [12]. Кроме того, такое повышение обладает потенциальными протективными эффектами в отношении воспалительной потери аксонов и способствует выживанию олигодендроцитов путем увеличения экспрессии IGF-1 (инсулиноподобного фактора роста 1) [13]. Однако дальнейший рост с суточными перепадами содержания в крови, зарегистрированный в нашем исследовании, по экспериментальным данным, способствует развитию неблагоприятных форм течения ЭАГ II стадии с потенциальным повреждением миокарда и головного мозга.

\section{ВЫВОДЫ}

Выявленные закономерности суточного ритма содержания LIF в крови больных ЭАГ ॥ стадии в виде увеличения в 20.00 на $15 \%$ и более с максимальным ростом в 2.00 ч на $22 \%$ и выше при сравнении с индивидуальным уровнем в 8.00 ч можно характеризовать как «патологические», а их сохранение на фоне гипотензивной терапии расценивать в качестве потенциального фактора прогредиентного течения гипертензии с повышением риска развития сердечно-сосудистых осложнений, несмотря на клинически благоприятное течение заболевания с достижением «целевого» АД. Представленные данные открывают перспективы дальнейшего изучения роли LIF с целью максимальной персонализации аналитических заключений изменения цитокина. Индивидуальный анализ суточных ритмов содержания в крови цитокинов, в частности LIF, является кандидатным диагностическим подходом для оценки скрытого прогрессирования заболевания при нормализации АД у пациента с эссенциальной гипертензией.

\section{Литература}

1. Mathieu M-E, Saucourt C, Mournetas V, Gauthereau X, Theze N, Praloran V, et al. LIF-Dependent Signaling: New Pieces in the Lego. Stem Cell Rev. 2012; 8 (1): 1-15.

2. Nicola NA, Babon JJ. Leukemia Inhibitory Factor (LIF). Cytokine \& growth factor reviews. 2015; 26 (5): 533-44.

3. Wu J, Xia S, Kalionis B, Wan W, Sun T. The Role of Oxidative Stress and Inflammation in Cardiovascular Aging. BioMed Research International. [Internet] 2014: 615312. Available from: https://doi.org/ 10.1155/2014.

4. González GE, Rhaleb N-E, D'ambrosio MA, Nakagawa P, Liu Y, Leung P, Dai X, Yang XP, Peterson EL, Carretero OA. Deletion of interleukin-6 prevents cardiac inflammation, fibrosis and dysfunction without affecting blood pressure in angiotensin II-high salt-induced hypertension. J Hypertens. 2015; (33): 144-52.

5. Bennardo M, Alibhai F, Tsimakouridze E, Chinnappareddy N, Podobed P, Reitz C, et al. Day-night dependence of gene expression and inflammatory responses in the remodeling murine heart post-myocardial. Am J Physiol Regul Integr Comp Physiol. 2016; 311 (6): 1243-1254

6. Чазова И. Е., Ратова Л. Г., Бойцов С. А., и др. Диагностика и лечение артериальной гипертонии. Системные гипертензии. 2010; (3): 5-26.

7. Zgheib C, Zouein FA, Kurdi M, Booz GW. Differential STAT3 Signaling in the Heart: Impact of Concurrent Signals and Oxidative

Stress. JAK-STAT. 2012; 1 (2): 101-10.

8. Fujio Y, Maeda M, Mohri T, Obana M, Iwakura T, Hayama A, et al. Glycoprotein 130 cytokine signal as a therapeutic target against cardiovascular diseases. J Pharmacol Sci. 2011; 117 (4): 213-22.

9. Радаева О. А., Симбирцев А. С. Изменение уровня фактора, ингибирующего лейкемию, в сыворотке периферической крови связано с прогрессированием эссенциальной артериальной гипертензии. Медицинский академический журнал. 2015; 15 (1): 34-42.

10. Zouein FA, Kurdi M, Booz GW. Dancing rhinos in stilettos: The amazing saga of the genomic and nongenomic actions of STAT3 in the heart. JAKSTAT. 2013; 2 (3): 101-10.

11. Jia D, Cai M, Xi Y, Du S, Zhenjun T. Interval exercise training increases LIF expression and prevents myocardial infarctioninduced skeletal muscle atrophy in rats. Life Sci. 2018; (193): 77-86.

12. Dey D, Shepherd A, Pachuau J, Migue M. Leukemia inhibitory factor regulates trafficking of T-type Ca2+ channels. Am J Physiol Cell Physiol. 2011; 300 (3): 576-87.

13. Xu L, Long J, Shi C, Zhang N, Lv Y, Feng J, et al. Effect of leukocyte inhibitory factor on neuron differentiation from human induced pluripotent stem cell-derived neural precursor cells. Int J Mol Med. 2018; 41 (4): 2037-49. https://doi.org/10.3892/ ijmm.2018.3418. 


\section{References}

1. Mathieu M-E, Saucourt C, Mournetas V, Gauthereau X, Theze N, Praloran V, et al. LIF-Dependent Signaling: New Pieces in the Lego. Stem Cell Rev. 2012; 8 (1): 1-15.

2. Nicola NA, Babon JJ. Leukemia Inhibitory Factor (LIF). Cytokine \& growth factor reviews. 2015; 26 (5): 533-44.

3. Wu J, Xia S, Kalionis B, Wan W, Sun T. The Role of Oxidative Stress and Inflammation in Cardiovascular Aging. BioMed Research International. [Internet] 2014: 615312. Available from: https://doi.org/ 10.1155/2014.

4. González GE, Rhaleb N-E, D'ambrosio MA, Nakagawa P, Liu Y, Leung P, Dai X, Yang XP, Peterson EL, Carretero OA. Deletion of interleukin-6 prevents cardiac inflammation, fibrosis and dysfunction without affecting blood pressure in angiotensin II-high salt-induced hypertension. J Hypertens. 2015; (33): 144-52.

5. Bennardo M, Alibhai F, Tsimakouridze E, Chinnappareddy N, Podobed P, Reitz C, et al. Day-night dependence of gene expression and inflammatory responses in the remodeling murine heart post-myocardial. Am J Physiol Regul Integr Comp Physiol. 2016; 311 (6): 1243-1254.

6. Chazova IE, Ratova LG, Bojcov SA, i dr. Diagnostika i lechenie arteria'noj gipertonii. Sistemnye gipertenzii. 2010; (3): 5-26. Russian.

7. Zgheib C, Zouein FA, Kurdi M, Booz GW. Differential STAT3 Signaling in the Heart: Impact of Concurrent Signals and Oxidative
Stress. JAK-STAT. 2012; 1 (2): 101-10.

8. Fujio Y, Maeda M, Mohri T, Obana M, Iwakura T, Hayama A, et al. Glycoprotein 130 cytokine signal as a therapeutic target against cardiovascular diseases. J Pharmacol Sci. 2011; 117 (4): 213-22.

9. Radaeva OA, Simbirtsev AS. Izmenenie urovnya faktora, ingibiruyushchego lejkemiyu, v syvorotke perifericheskoj krovi svyazano s progressirovaniem essencialnoj arterialnoj gipertenzii. Medicinskij akademicheskij zhurnal. 2015; 15 (1): 34-42. Russian.

10. Zouein FA, Kurdi M, Booz GW. Dancing rhinos in stilettos: The amazing saga of the genomic and nongenomic actions of STAT3 in the heart. JAKSTAT. 2013; 2 (3): 101-10.

11. Jia $\mathrm{D}$, Cai $M, X i \mathrm{Y}$, Du $\mathrm{S}$, Zhenjun $\mathrm{T}$. Interval exercise training increases LIF expression and prevents myocardial infarctioninduced skeletal muscle atrophy in rats. Life Sci. 2018; (193): 77-86.

12. Dey D, Shepherd A, Pachuau J, Migue M. Leukemia inhibitory factor regulates trafficking of T-type Ca2+ channels. Am J Physiol Cell Physiol. 2011; 300 (3): 576-87.

13. Xu L, Long J, Shi C, Zhang N, Lv Y, Feng J, et al. Effect of leukocyte inhibitory factor on neuron differentiation from human induced pluripotent stem cell-derived neural precursor cells. Int J Mol Med. 2018; 41 (4): 2037-49. https://doi.org/10.3892/ ijmm.2018.3418. 